\title{
A gender study of food stress and implications for international students acculturation
}

Quan-Hoang Vuong ${ }^{1}$, Minh-Hoang Nguyen ${ }^{1}$, Quang-Loc Nguyen ${ }^{2}$, Quang-Trung Nguyen ${ }^{3}$, and Tam-Tri Le ${ }^{1, *}$

${ }^{1}$ Centre for Interdisciplinary Social Research, Phenikaa University, Yen Nghia Ward, Ha Dong District, Hanoi 100803, Vietnam

2 SP Jain School of Global Management, New South Wales 2141, Australia

${ }^{3}$ A.I. for Social Data Lab (AISDL), Vuong \& Associates, Hanoi 100000, Vietnam

* Corresponding: tri.letam@phenikaa-uni.edu.vn

(Un-peer-reviewed manuscript v1)

23 October 2021 


\begin{abstract}
Acculturative stress can be a big problem for international students. Among all the difficulties they may face, adjusting to new foods in a new environment is crucial to their well-being. Research related to food acculturation is limited but also hints at gender differences. Using the Bayesian analysis approach with the Hamiltonian MCMC (Markov Chain Monte Carlo) technique on a sample of 268 students from a Japanese international university, we found that female students are more likely to have perceived difficulties in the process of adjusting to new foods, but social connectedness lessens this effect. We also found no significant differences between domestic and international students regarding perceived difficulties on food adjustment in this highly multicultural study site. We suggest international universities provide better information about the food situations on campuses, especially for female students, and organize more cultural exchange events and food-related social activities to help students overcome barriers of food stress.
\end{abstract}

Keywords: food acculturation; gender difference; acculturative stress; international student; multicultural environment

\title{
1. Introduction
}

Our world is becoming increasingly interconnected. With the rapid advancement in transportation and information technology, globalization is now an important property in various aspects of human society, such as the economy, education, and culture. People living in a new environment will need to go through psychological adjustments related to their perceptions of new cultural values, which is the process of acculturation (John W. Berry, 2005). In terms of information processing, people during the acculturative process will receive and evaluate new values (as well as re-evaluate stored old values) to decide what to accept and what to reject as a natural mechanism to update one's mindset (Q. H. Vuong, 2016; Q. H. Vuong \& Napier, 2015). This process is often not easy and may cause acculturative stress to certain degrees (John W Berry, 2006; John W Berry \& Annis, 1974; John W Berry, Kim, Minde, \& Mok, 1987). Acculturative stress was found to be positively associated with depression (Park \& Rubin, 2012; Revollo, Qureshi, Collazos, Valero, \& Casas, 2011; Tummala-Narra, Alegria, \& Chen, 2012), and notedly among international students (Constantine, Okazaki, \& Utsey, 2004; M. H. Nguyen, Le, \& Meirmanov, 2019). Understanding the difficulties people may face during the acculturative process, including food stress, can help create better support strategies to minimize negative effects.

Food is an essential part of life, not only from the angle of nutrition and physical well-being relationship but also from the perspective that cuisine is a major aspect of human culture. Each culture perceives food items' social, spiritual, and emotional well-being qualities differently (Ares et al., 2016). Coming to a new environment, people will need to adjust to new food and set up their nutrition plans accordingly. Overall, food acculturation is a complex problem, yet research on the topic is still limited.

The process of food acculturation can be difficult, where many different internal and external factors can influence how people adapt to new foods, which points to the importance of social support to help people familiarize themselves with foreign cuisine cultures (Hartwell, Edwards, \& 
Brown, 2011). It is also suggested that over time, food acculturation may lead to a shift into new eating habits different from one's original diet - which, in the studied case, is the dominance of the host country's characteristics (Batis, Hernandez-Barrera, Barquera, Rivera, \& Popkin, 2011). Regardless of adaptation strategies, the changes in perceptions and behaviors toward cultural values within a multicultural environment are under the phenomenon of cultural additivity on both individual and social levels (Q.-H. Vuong et al., 2018). Thus, examining the social factors affecting the food acculturation process with the help of an information processing framework for cognitive changes can provide helpful insights.

Regarding gender differences in food perception, studies have found that women are often more concerned about the health impacts and weight control aspects of food choice (Westenhoefer, 2005). Additionally, women tend to care more about their food's "virtuous" qualities across dimensions such as health, related ethical issues, and body image (Beardsworth et al., 2002). Regarding gender differences in acculturation, studies on international students in Japan found that females are at higher risk of depression (Eskanadrieh et al., 2012; M. H. Nguyen et al., 2019). A study on food patterns of Korean immigrants in the United States found that women are less likely to be acculturated and less likely to be overweight than men (Jasti, Lee, \& Doak, 2011).

In this present investigation, we examine the gender difference in the perceived difficulty of food acculturation. As social connectedness is a predictor of acculturative stress level (Yeh \& Inose, 2003), its influence in terms of information processing mechanism can help better explain the adjustment process, including the assumed gender difference. Thus, we conducted the present study using the Bayesian Mindsponge Framework (BMF) on a sample of 268 students from a Japanese international university. The campus of this university is a highly multicultural environment (see the Study Site and Participants subsection below for more details), which also helps investigate the food adjustment issues among both domestic and international students (see the Model Construction subsection below). It is important to note that the data used in this current study were collected in 2018 - before the COVID-19 pandemic - and all activities on the campus were normal.

\section{Materials and Methods}

\subsection{Model Construction}

We used the Bayesian Mindsponge Framework (BMF, also known as Bayesian Mindsponge analytical approach) to explain and analyze the acculturative process of adjusting to new food (M.-H. Nguyen \& Le, 2021; M.-H. Nguyen et al., 2021). In detail, the Mindsponge mechanism (Q. H. Vuong, 2016; Q. H. Vuong \& Napier, 2015) was used as the theoretical foundation for model construction, while Bayesian inference was utilized to fit the model. The Mindsponge mechanism is an information processing framework of receiving, filtering, and accepting (or rejecting) new values, which can be used to explain acculturative processes. In a new (and multicultural) environment, students need to absorb and evaluate new values to adjust their mindset accordingly, which is necessary for them to continue living there through adaptation (regardless of chosen strategies). This framework was used to provide insights on how acculturative stress and social connectedness may affect university students' depression levels (M. H. Nguyen et al., 2019). 
In the Mindsponge mechanism, there are two basic conditions for the mind to incorporate a new value: information accessibility (the mind can receive the information that carries the value) and favorable evaluation (subjective cost-benefit judgments based on existing trusted values in the mindset resulting in a positive assessment of that value) (M.-H. Nguyen et al., 2021; Q.-H. Vuong, Nguyen, \& Le, 2021a). The mindset updated with new trusted (accepted) values will affect the subsequent evaluation of similar values in the respective direction - such updating manner is what gradually creates adaptation. This Mindsponge mechanism is naturally present in any acculturative process.

In the current investigation, the new value we focus on is new foods, and the adjustment to them in this context is an acculturative process. Many factors with complex interactions affect how students adjust to new food during their university years in the new environment. Such factors can be: personal food preference, food price, food availability, preparation (cooking) ability, health concerns, as well as many other social factors (curiosity, eating with friends, cultural exchange, etc.). Regardless of the complex influences, we can test the overall probabilities as follows. First, we want to know if there is any major difference between domestic and international students regarding perceived food adjustment difficulties in the studied campus's highly multicultural environment (see the Study site sub-section below). Next, we focus on examining the gender-based difference, but we also want to know the moderating factor in terms of information processing. Social connectedness has been shown to function as a "priority pass" that facilitates the processes of receiving and filtering new information in the Mindsponge mechanism (M.-H. Nguyen et al., 2021; Q.-H. Vuong, Nguyen, \& Le, 2021b). Quickly assessing a lot of related information can help ease the perceived difficulties in a new environment during the food adjustment process. Based on the reasoning above, we derive the following model.

$$
\text { FoodProblem } \sim \alpha+\text { Inter_Dom }+ \text { Gender }+ \text { Gender } * \text { SocialConnectedness }
$$

\subsection{Study Site and Participants}

This study employed a dataset from the survey on students' mental health and social connectedness in a multicultural university campus. The data collection was conducted in Ritsumeikan Asia Pacific University (APU), an international university in Oita Prefecture, Japan, from October to December 2018. The students were from 86 countries and regions, and faculty members were from 22 countries and regions at the time of survey collection. International students accounted for around $50.1 \%$ of the whole university's students on campus during the survey period. The data has been employed and validated in several research papers (M.-H. Nguyen, Ho, Nguyen, \& Vuong, 2019; M.-H. Nguyen et al., 2021; M.-H. Nguyen, Serik, Vuong, \& Ho, 2019; M. H. Nguyen et al., 2019).

There were $75 \%$ international students and $25 \%$ domestic ones in the total sample of 268 students. Percentages of female and male students were $63 \%$ and $37 \%$, respectively. The origins of international students were diverse, ranging from East Asia, Sought East Asia to America. Among all regions, the majority of students were from South East Asia, accounting for $45.5 \%$ of the total international students. The complete description and validation of the dataset can be viewed in the article "A Dataset of Students' Mental Health and Help-Seeking Behaviors in a Multicultural Environment" (M.-H. Nguyen, M.-T. Ho, et al., 2019). The dataset is openly 
accessible online, which helps with transparent and cost-effective scientific cross-checking or reproduction (Q.-H. Vuong, 2018).

The survey procedure strictly conformed to the World Medical Association (WMA) Declaration of Helsinki and was permitted by APU \& Ethical Committee Board Approval Number 2018-03. Before participants filled in the questionnaire, the survey's purpose, content, confidentiality, and the right to refuse to participate in the survey were clearly explained.

\subsection{Variable description}

Within the dataset, we purposely chose variables that can help explain the acculturative process regarding adjusting to new food among international and domestic students. In total, we employed four variables. The description of each variable is presented in Table 1.

Table 1. Variable description.

\begin{tabular}{|c|c|c|c|}
\hline Name & Variable & $\begin{array}{l}\text { Type of } \\
\text { variable }\end{array}$ & Description \\
\hline $\begin{array}{l}\text { Feeling } \\
\text { uncomfortable in } \\
\text { adjusting to new } \\
\text { foods }\end{array}$ & FoodProblem & Continuous & $\begin{array}{l}\text { The score of having stress when } \\
\text { encountering new foods. The data } \\
\text { range from } 1 \text { (low) to } 5 \text { (high). }\end{array}$ \\
\hline $\begin{array}{c}\text { Social } \\
\text { connectedness }\end{array}$ & SocialConnectedness & Continuous & $\begin{array}{c}\text { The total score of social } \\
\text { connectedness ( } 8 \text { items) was } \\
\text { measured by the Social } \\
\text { Connectedness Scale developed by } \\
\text { Lee and Robins (R. M. Lee \& } \\
\text { Robbins, } 1995 \text { ). The original values } \\
\text { range from } 8 \text { to } 48 \text {, then divided by } \\
\text { the total item number to better fit the } \\
\text { model. }\end{array}$ \\
\hline Gender of student & Gender & Binominal & $\begin{array}{l}\text { Gender of students: Male or Female. } \\
\text { 'Female student' is coded as } 1 \text {, 'Male } \\
\text { student' is coded as } 0 .\end{array}$ \\
\hline Type of student & Inter_Dom & Binominal & $\begin{array}{l}\text { Type of student: International student } \\
\text { (Inter) or domestic one (Dom). } \\
\text { 'International student' is coded as } 1 \text {, } \\
\text { 'Domestic student' is coded as } 0 .\end{array}$ \\
\hline
\end{tabular}

We generated an additional variable, Gender_SocialConnectedness, besides the above variables (Table 1) from the dataset. The variable Gender_SocialConnectedness is the interaction between two variables, Gender and SocialConnectedness, used to examine their influence on FoodProblem. The outcome variable FoodProblem was based on the answers to the question "I feel uncomfortable to adjust to new foods". Respondents choose one in five response options: "Strongly disagree", "Disagree", "Not sure", "Agree", "Strongly agree". These responses were coded as 1, 2, 3, 4, and 5, respectively. The question was based on the Acculturative Stress Scale for International Students proposed by Sandhu and Asrabadi (1994). 
The students' social connectedness is determined by asking eight questions about feelings of connection to others in their social environment, demonstrated by the SocialConnectedness variable. The social connectedness level was rated based on a 6-point Social Connectedness Scale ranging from 1 ('Strongly disagree') to 6 ('Strongly agree'), developed by Lee and Robins (R. M. Lee \& Robbins, 1995). The two demographic variables, Gender and Inter_Dom, are determined by asking respondents, "What is your gender?" and "Are you a domestic student or an international student?" respectively.

\subsection{Statistical Analysis}

For assessing the acculturative food issue in students, the data were analyzed using the Bayesian analysis method with the Hamiltonian MCMC (Markov Chain Monte Carlo) technique. There are three main reasons for employing the Bayesian method in this study. First, since the evidence in the current study is novel, the Bayesian's updating capability can benefit future similar studies (McElreath, 2018; Wagenmakers et al., 2018). Second, since the Bayesian inference approach treats all the properties probabilistically, including unknown parameters, it aids the construction of parsimonious models (Wagenmakers et al., 2018). By incorporating the power of the MCMC technique, Bayesian methods can fit a wide variety of models, like multilevel correlation structures and non-linear regression frameworks, making flexibility a major advantage (Dunson, 2001). Finally, since the sample size in the dataset is small, the Bayesian MCMC simulation technique also helps enhance the estimation's precision (Uusitalo, 2007). Given these features, the Bayesian MCMC approach is highly compatible with the Mindsponge framework, and thus, the BMF was employed.

The data were analyzed using $\mathrm{R}$ software (version 4.0.2) with the bayesvl package (Q.- $\mathrm{H}$. Vuong, La, Nguyen, Ho, Ho, et al., 2020; Q.-H. Vuong, La, Nguyen, Ho, Tran, et al., 2020). The package has been employed in several psychological studies using BMF (M.-H. Nguyen et al., 2021; Q.-H. Vuong et al., 2021a, 2021b) due to its good visualization power and user-friendly, transparent operation. The analysis was run using 5000 iterations (2000 are warm-up iterations), four Markov chains, and four cores. To check the model's goodness-of-fit, we employed the Pareto smoothed importance-sampling leave-one-out cross-validation (PSIS-LOO) approach (Vehtari, Gelman, \& Gabry, 2017). We checked the Markov property with effective sample sizes, Gelman factors, and several visualized plots (see Results section). Because this is the first study of its scope and method, all estimations' priors were set as uninformative (McElreath, 2018), equivalent to a normal distribution with mean $=0$ and standard deviation $=10$.

\section{Results}

To test the hypothesis, we examined the model as presented in the Model Construction subsection. The model examined the effects of students' gender and its interaction with social connectedness, the type of student (international or domestic student) on the perceived difficulty of adjusting to new food. The model's logical network is shown in Figure 1. 


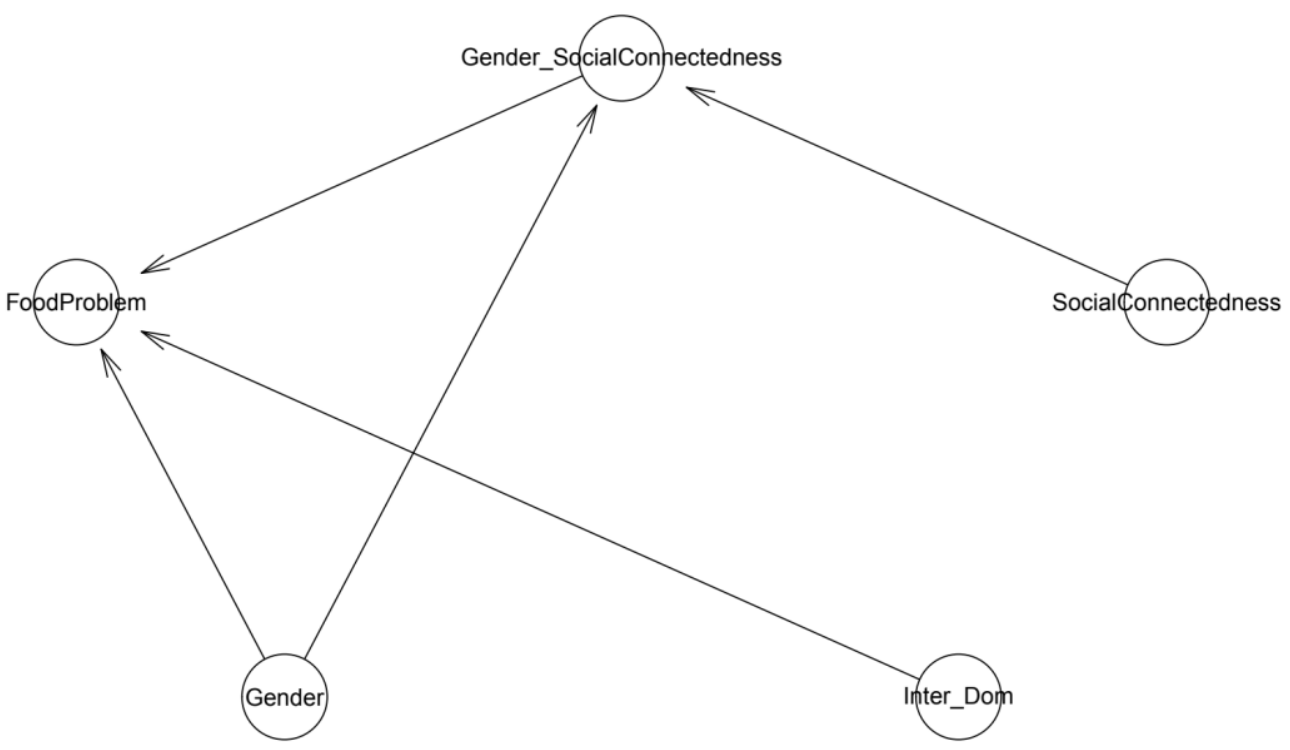

Figure 1: the model's logical network

The PSIS diagnostic plot shows that all $\mathrm{k}$ values are below 0.5 , implying that the model has a high goodness-of-fit with the data (see Figure 2). 


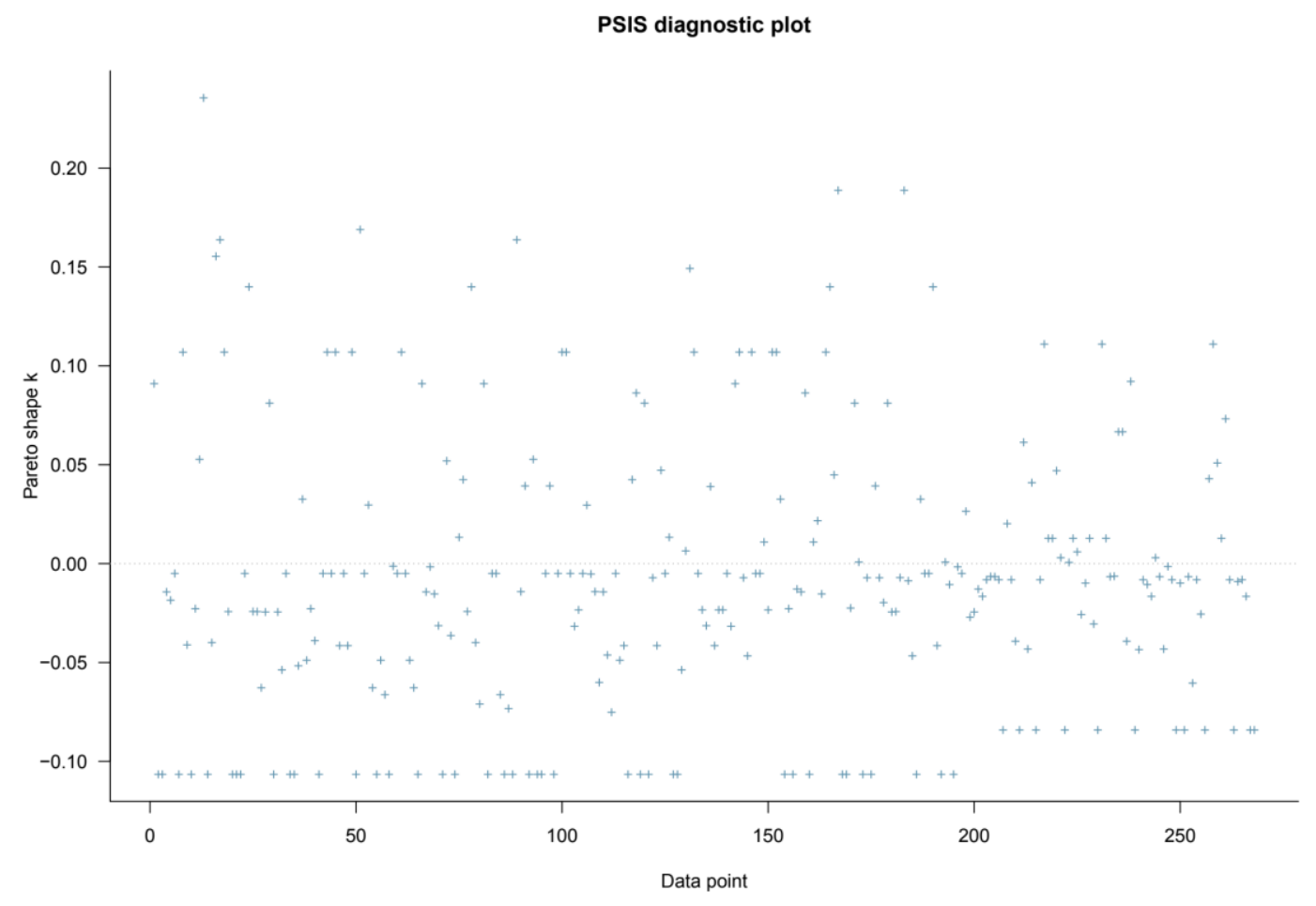

Figure 2: the model's PSIS diagnostic plot

All parameters show a good convergence since the effective sample sizes ( $n$ _eff) are greater than 1000 and Gelman shrink factors (Rhat) all equal one (see Table 2). Furthermore, the convergence is also confirmed visually using trace plots, Gelman plots, and autocorrelation plots (see Figure 3,4,5).

Table 2: the model's simulated posteriors

\begin{tabular}{|c|c|c|c|c|}
\hline Parameters & Mean & $\begin{array}{c}\text { Standard } \\
\text { Deviation }\end{array}$ & N_eff & Rhat \\
\hline FoodProblem & 1.75 & 0.14 & 5959 & 1 \\
\hline Inter_Dom & -0.04 & 0.13 & 6347 & 1 \\
\hline Gender & 1.03 & 0.30 & 5730 & 1 \\
\hline Gender_SocialConnectedness & -0.19 & 0.06 & 5793 & 1 \\
\hline
\end{tabular}

The trace plots in Figure 3 show the Markov chains fluctuating around a central equilibrium (posterior values on the $y$-axis over iterations on the $x$-axis), indicating that the chains converge well. 

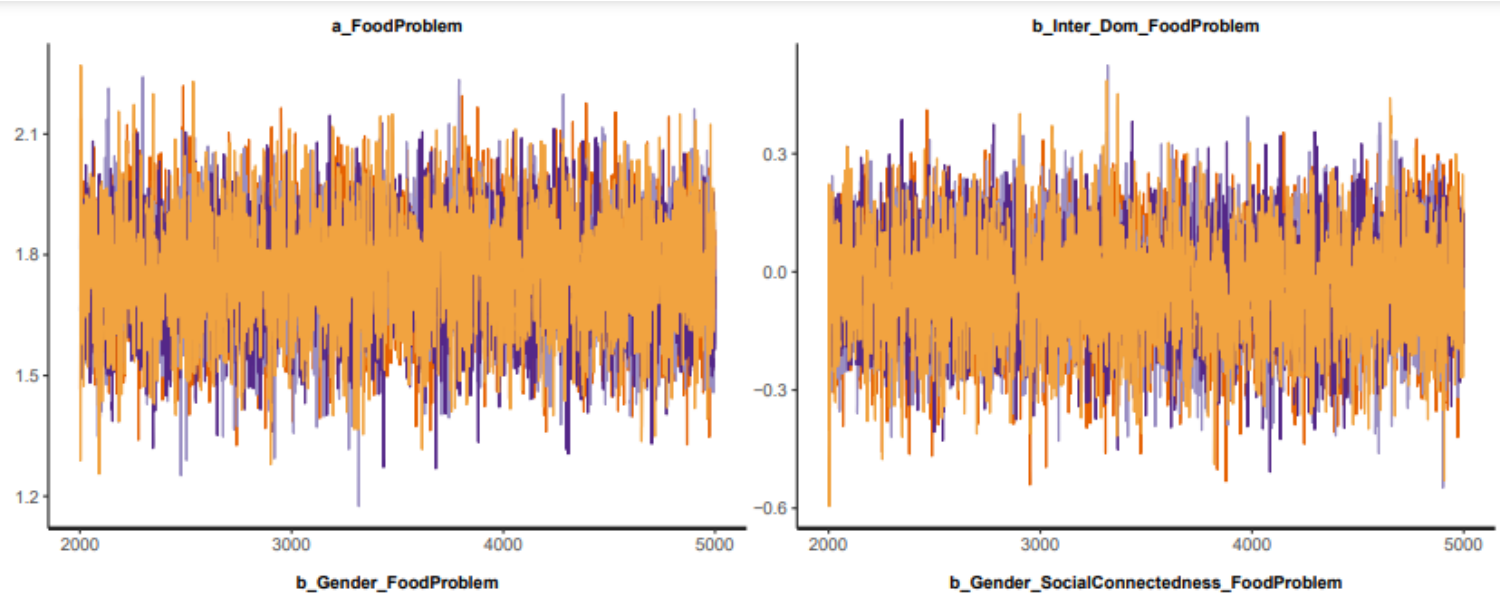

chain
-1
-2
-3
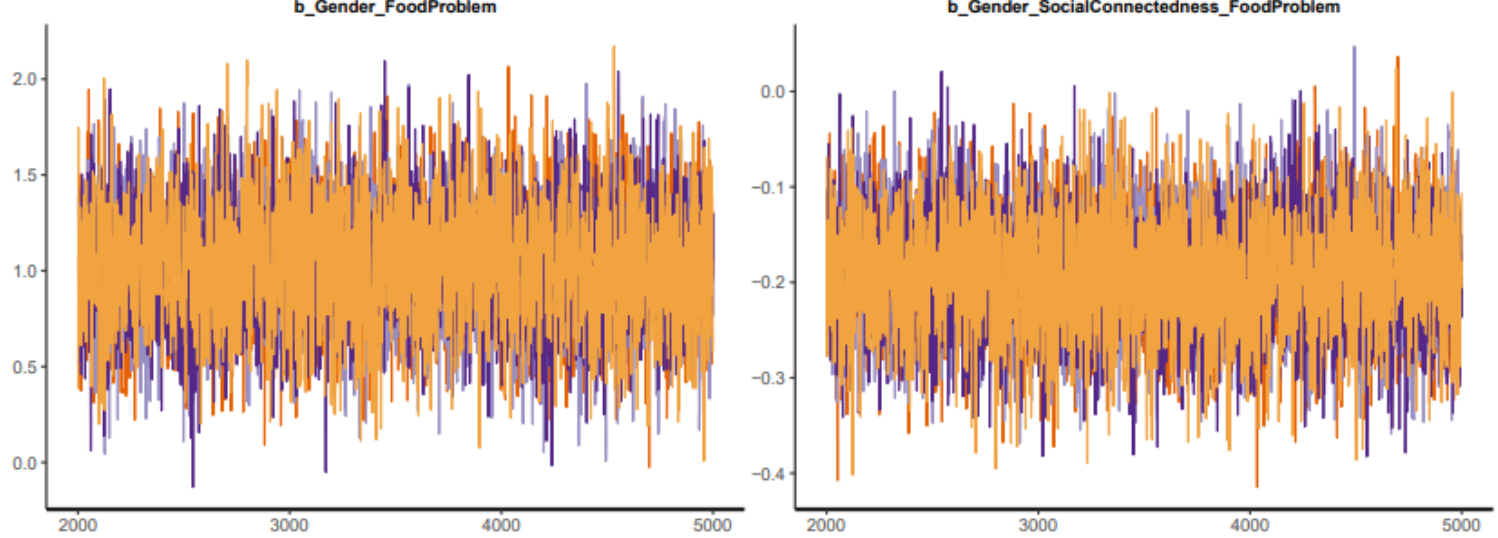

Figure 3. Trace plots for the model's posterior coefficients

As shown in Figure 4, the shrink factors rapidly drop to one during the warm-up phase, meaning that the average variance between chains and the average variance within chains is similar, which indicates good convergence (Gelman \& Rubin, 1992). Additionally, we can observe in Figure 5 that the autocorrelation for each parameter is quickly diminishing, which again confirms the healthy Markov property of the chains. 

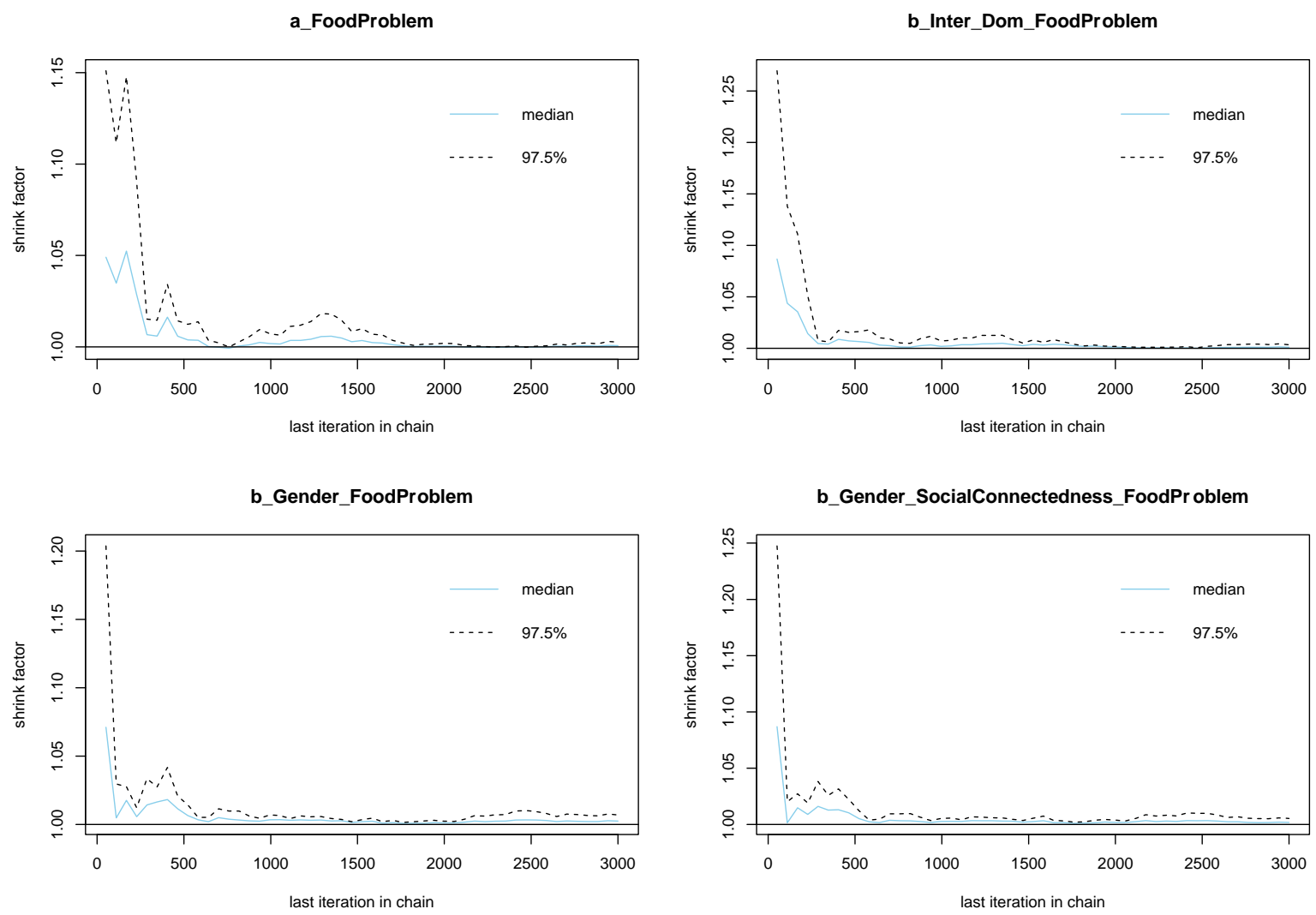

Figure 4. Gelman plots for the model's posterior coefficients. 

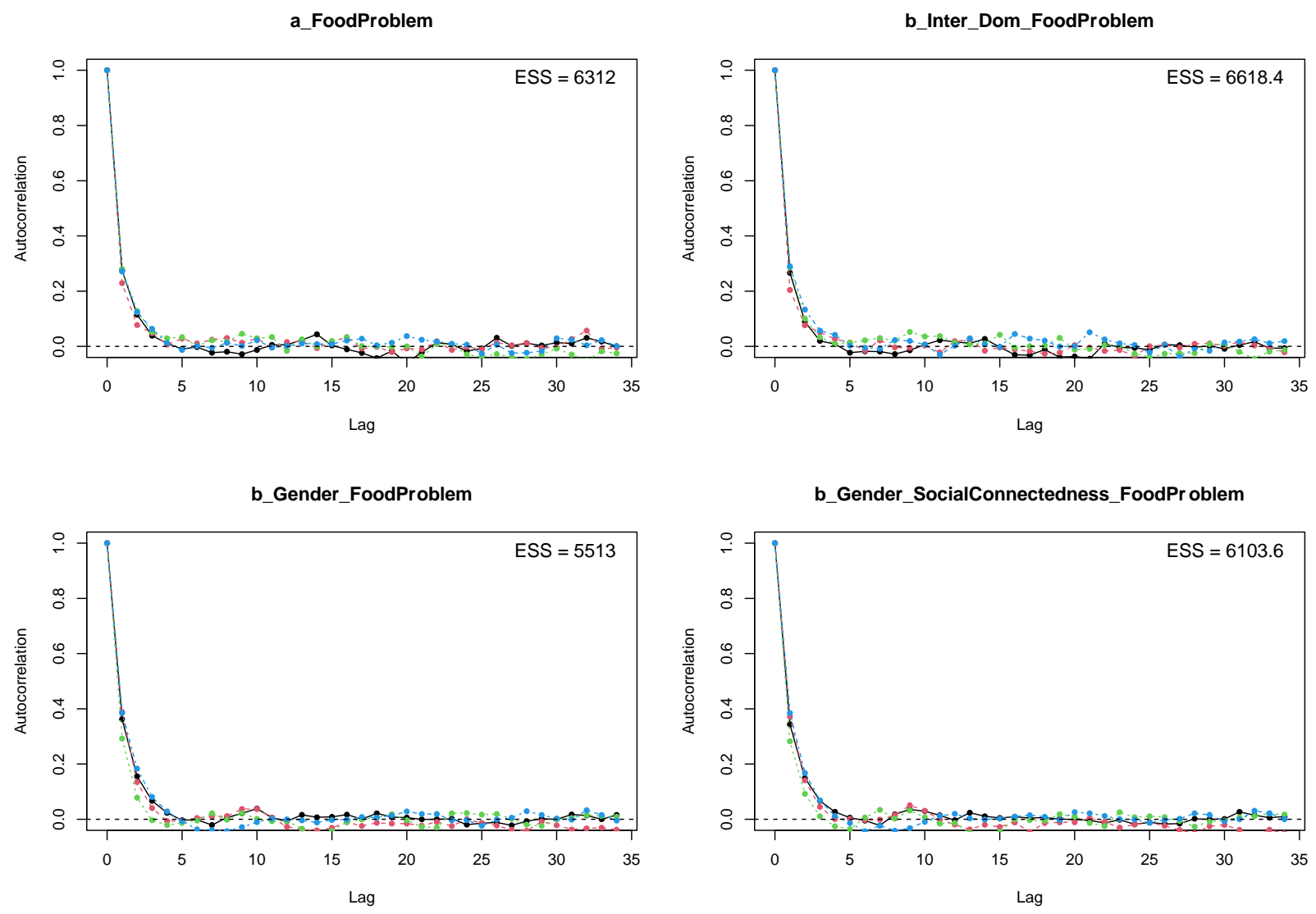

Figure 5. Autocorrelation plots for the model's posterior coefficients.

Regarding gender, females are found to be positively associated with a higher level of perceived difficulties in adjusting to new food $\left(\mu_{\text {Gender }}=1.03\right.$ and $\left.\sigma_{\text {Gender }}=0.30\right)$. However, the interaction with social connectedness produces a negative value, suggesting social connectedness lessens the effect of gender on food problems $\left(\mu_{\text {Gender_Socialconnectedness }}=-0.19\right.$ and $\sigma_{\text {Gender_socialConnectedness }}=0.06$ ). The impact of the type of student (domestic or international student) is found to be not significant toward food acculturative problems $\left(\mu_{\text {Inter_Dom }}=-0.04\right.$ and $\sigma_{\text {Inter_Dom }}=0.13$ ). The posterior distributions of Gender and Gender_SocialConnectedness fall entirely on the positive and negative, respectively, indicating high reliability of the results (see Figure 6). We can also see that the majority of simulated samples are located on the positive side for Gender (x-axis) and the negative side for Gender_SocialConnectedness (y-axis) in the pairwise distribution graph (see Figure 7). 


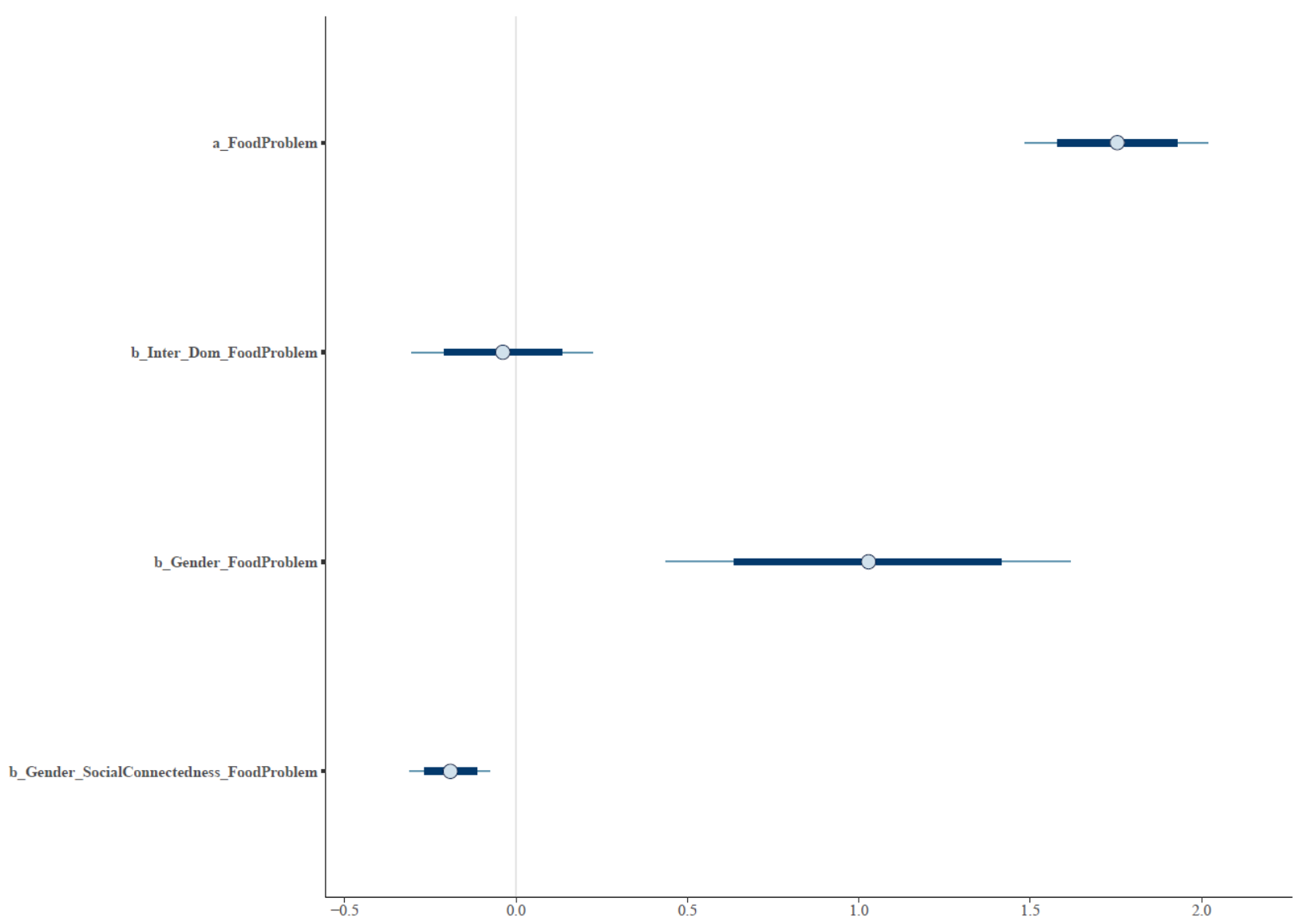

Figure 6. Interval distributions of the model's posterior coefficients. 


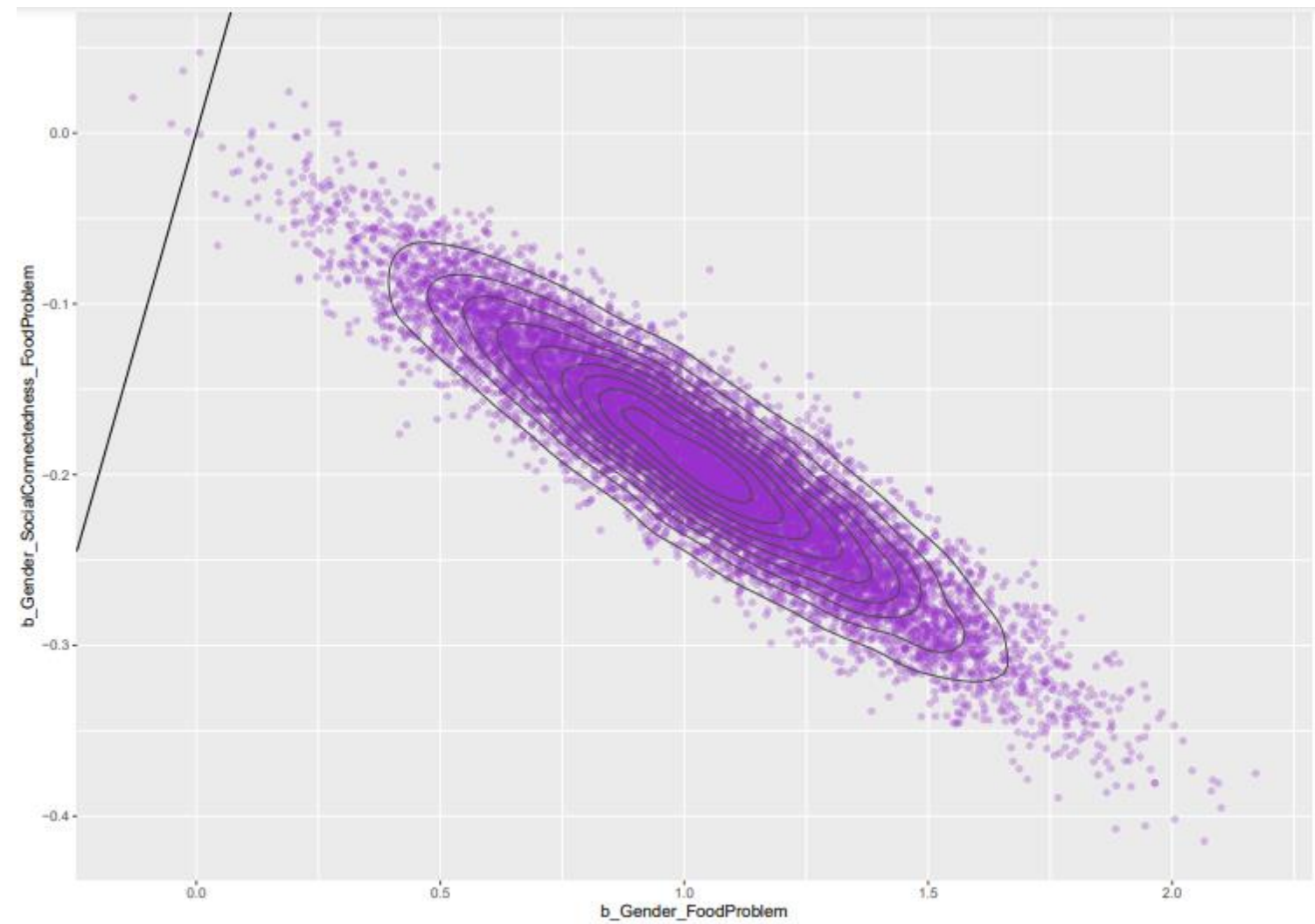

Figure 7. Pairwise distribution plot for Gender and Gender_SocialConnectedness.

\section{Discussion}

Our analysis result shows no significant difference between domestic and international students regarding the perceived difficulties in adjusting to new food. While some former studies only focused on examining the acculturative effects in international students or immigrants coming to the host countries (Batis et al., 2011; Hartwell et al., 2011), our study investigates both domestic and international students due to the specific characteristics of the study site (APU). To elaborate, at the time of data collection, about half of the total student population on campus were international students (see the Methodology section). APU regularly held social events (such as Multicultural Weeks) co-organized by students to introduce and exchange the cultural values of every country or region (APU, 2021). Foods from various cultures are accessible to all students not only during these events but also by the school cafeteria's diverse menu as well as many independent food vendors on the campus. There are also shared cooking activities among domestic and international students in the university's dormitory. Additionally, the local government has been supporting cultural diversity, and a wide range of foreign food items are available in non-Japanese restaurants and supermarkets across the town. In this highly multicultural environment, all students, including domestic ones, need to adjust to the new situation regarding food; thus, the acculturative process can be applied to all students regardless of their origins.

Regarding gender differences, we found that female students are more likely to perceive difficulties in the food acculturation process; however, a higher level of social connectedness helps lessen this effect. Several characteristics can possibly explain why female students may 
find it harder to adjust to new food. Women are selective regarding food choice due to a higher level of concern about the nutrition, body image, and ethical implication (Beardsworth et al., 2002; Westenhoefer, 2005). As women may exhibit stronger reactions to stressors (Misra, Crist, \& Burant, 2003), their concerns about food choice can increase difficulties adapting to diet changes. Additionally, women may be more risk-averse than men (Charness \& Gneezy, 2012), making them hesitant to try new foods and nutrition plans. Another study suggested that this risk-aversion attitude is self-assessed (Brighetti \& Lucarelli, 2015), but subjective assessments would still affect the ideation of perceived risks.

How does social connectedness help female students better cope with food adjustment? Women may perceive a lower level of social support than men during the acculturative process (Allen, Amason, \& Holmes, 1998). Still, they may also rely more on collectivistic approaches in dealing with acculturative stress (D. S. Lee \& Padilla, 2014). This suggests that a stronger feeling of being connected to other people in the society can help female students find the adaptation less stressful.

In terms of information processing mechanism, a sense of connectedness was shown to have the role of a "priority-pass" that speed up the process of receiving and filtering information, increasing the probability of external information absorption and the acceptance of values into one's mindset (M.-H. Nguyen et al., 2021). Adaptation requires a lot of information from many sources as well as evaluating such information. In this case, they are food-related information such as taste, nutritional properties, availability, price, attached cultural and ethical values, etc. Adjusting to new diets in a new living environment is not as simple as trying the items to see if one likes it or not, but rather is a complex assessment based on corresponding perceived values of various aspects (a Mindsponge process of adaptation). Therefore, a higher level of social connectedness will make it easier to gather information from social groups or ask for advice from other people about food. The willingness to connect socially also increases trust toward the information sources, which helps understand and accept the attached values of new food items.

These results have several implications for policy on acculturation and international education. In a highly multicultural environment, everyone is exposed to new cultural values. As the world is becoming more globalized, managing international environments for education, business, or living requires careful consideration of the effects of acculturation on all population groups. For international university campuses, besides a sufficient supply of various food items from many cultures, more specific information about food situations (variety, availability, etc.) at the living environment (campus and local places) should be provided to students in advance. And since gender inequality issues are still a big concern in the globalization context (Kanbur, 2015), our study shows that there needs to be more support for female students regarding acculturationrelated difficulties. Here, providing professional advice about the nutritional values, health impacts, diet structures, and ethical implications of new foods can help female students assess their related concerns quicker and healthier. Social activities such as cultural exchange events or cooking and eating together can increase the level of social connectedness among students in the new environment, helping them provide each other with information and practical support.

The current study's limitations are presented here for transparency (Q.-H. Vuong, 2020). The sample we use for analysis is small and has a higher female to male ratio. However, we employed the Bayesian MCMC approach to help increase the simulated results' precision. Additionally, the number of APU international students with Asian origins is higher than those 
from other regions; thus, this multicultural environment may not represent all cultures equally sufficiently.

\section{References}

Allen, M. W., Amason, P., \& Holmes, S. (1998). Social support, Hispanic emotional acculturative stress and gender. Communication Studies, 49(2), 139-157. doi:10.1080/10510979809368525

APU. (2021). Multicultural Weeks - Ritsumeikan Asia Pacific University. Retrieved from https://en.apu.ac.jp/home/life/content25/

Ares, G., Giménez, A., Vidal, L., Zhou, Y., Krystallis, A., Tsalis, G., . . . Deliza, R. (2016). Do we all perceive food-related wellbeing in the same way? Results from an exploratory cross-cultural study. Food Quality and Preference, 52, 62-73. doi:10.1016/j.foodqual.2016.03.014

Batis, C., Hernandez-Barrera, L., Barquera, S., Rivera, J. A., \& Popkin, B. M. (2011). Food Acculturation Drives Dietary Differences among Mexicans, Mexican Americans, and Non-Hispanic Whites. The Journal of Nutrition, 141(10), 1898-1906. doi:10.3945/jn.111.141473

Beardsworth, A., Bryman, A., Keil, T., Goode, J., Haslam, C., \& Lancashire, E. (2002). Women, men and food: the significance of gender for nutritional attitudes and choices. British Food Journal, 104(7), 470-491. doi:10.1108/00070700210418767

Berry, J. W. (2005). Acculturation: Living successfully in two cultures. International Journal of Intercultural Relations, 29(6), 697-712. doi:10.1016/j.ijintrel.2005.07.013

Berry, J. W. (2006). Acculturative stress. In P. T. P. Wong \& L. C. J. Wong (Eds.), Handbook of multicultural perspectives on stress and coping (pp. 287-298). Boston (MA): Springer.

Berry, J. W., \& Annis, R. C. (1974). Acculturative stress: The role of ecology, culture and differentiation. Journal of Cross-Cultural Psychology, 5(4), 382-406.

Berry, J. W., Kim, U., Minde, T., \& Mok, D. (1987). Comparative studies of acculturative stress. International Migration Review, 21(3), 491-511.

Brighetti, G., \& Lucarelli, C. (2015). Gender differences in attitudes towards risk and ambiguity: when psycho-physiological measurements contradict sex-based stereotypes. International Journal of Entrepreneurship and Small Business, 24(1), 62. doi:10.1504/IJESB.2015.066153

Constantine, M. G., Okazaki, S., \& Utsey, S. O. (2004). Self-Concealment, Social Self-Efficacy, Acculturative Stress, and Depression in African, Asian, and Latin American International College Students. American Journal of Orthopsychiatry, 74(3), 230-241. doi:10.1037/0002-9432.74.3.230

Dunson, D. B. (2001). Commentary: Practical Advantages of Bayesian Analysis of Epidemiologic Data. American Journal of Epidemiology, 153(12), 1222-1226. doi:10.1093/aje/153.12.1222

Eskanadrieh, S., Liu, Y., Yamashina, H., Kono, K., Arai, A., Lee, R. B., \& Tamashiro, H. (2012). Depressive symptoms among international university students in northern Japan: Prevalence and associated factors. Kokusai Hoken Iryo (Journal of International Health), 27(2), 165-170. doi:10.11197/jaih.27.165

Gelman, A., \& Rubin, D. B. (1992). Inference from Iterative Simulation Using Multiple Sequences. Statistical Science, 7(4), 457-472. doi:10.1214/ss/1177011136

Hartwell, H. J., Edwards, J. S. A., \& Brown, L. (2011). Acculturation and food habits: lessons to be learned. British Food Journal, 113(11), 1393-1405. doi:10.1108/00070701111180003

Jasti, S., Lee, C. H., \& Doak, C. (2011). Gender, Acculturation, Food Patterns, and Overweight in Korean Immigrants. American Journal of Health Behavior, 35(6), 734-745. doi:10.5993/AJHB.35.6.9

Kanbur, R. (2015). Globalization and Inequality. In A. B. Atkinson \& F. Bourguignon (Eds.), Handbook of Income Distribution (Vol. 2, pp. 1845-1881). Amsterdam: Elsevier. 
Lee, D. S., \& Padilla, A. M. (2014). Acculturative Stress and Coping: Gender Differences Among Korean and Korean American University Students. Journal of College Student Development, 55(3), 243262. doi:10.1353/csd.2014.0025

Lee, R. M., \& Robbins, S. B. (1995). Measuring belongingness: The social connectedness and the social assurance scales. Journal of counseling psychology, 42(2), 232.

McElreath, R. (2018). Statistical rethinking: A Bayesian course with examples in R and Stan. Boca Raton, London, New York: Chapman and Hall/CRC Press.

Misra, R., Crist, M., \& Burant, C. J. (2003). Relationships Among Life Stress, Social Support, Academic Stressors, and Reactions to Stressors of International Students in the United States. International Journal of Stress Management, 10(2), 137-157. doi:10.1037/1072-5245.10.2.137

Nguyen, M.-H., Ho, M.-T., Nguyen, Q.-Y. T., \& Vuong, Q.-H. (2019). A Dataset of Students' Mental Health and Help-Seeking Behaviors in a Multicultural Environment. Data, 4(3), 124. doi:10.3390/data4030124

Nguyen, M.-H., \& Le, T.-T. (2021). Bayesian Mindsponge Framework. In Scholarly Community Encyclopedia. Basel, Switzerland: MDPI AG.

Nguyen, M.-H., Le, T.-T., Nguyen, H.-K. T., Ho, M.-T., Nguyen, H. T. T., \& Vuong, Q.-H. (2021). Alice in Suicideland: Exploring the Suicidal Ideation Mechanism through the Sense of Connectedness and Help-Seeking Behaviors. International Journal of Environmental Research and Public Health, 18(7), 3681. doi:10.3390/ijerph18073681

Nguyen, M.-H., Serik, M., Vuong, T.-T., \& Ho, M.-T. (2019). Internationalization and Its Discontents: HelpSeeking Behaviors of Students in a Multicultural Environment Regarding Acculturative Stress and Depression. Sustainability, 11(7), 1865. doi:10.3390/su11071865

Nguyen, M. H., Le, T. T., \& Meirmanov, S. (2019). Depression, Acculturative Stress, and Social Connectedness among International University Students in Japan: A Statistical Investigation. Sustainability, 11(3), 878. doi:10.3390/su11030878

Park, H.-S., \& Rubin, A. (2012). The mediating role of acculturative stress in the relationship between acculturation level and depression among Korean immigrants in the U.S. International Journal of Intercultural Relations, 36(5), 611-623. doi:10.1016/j.ijintrel.2012.04.008

Revollo, H.-W., Qureshi, A., Collazos, F., Valero, S., \& Casas, M. (2011). Acculturative stress as a risk factor of depression and anxiety in the Latin American immigrant population. International Review of Psychiatry, 23(1), 84-92. doi:10.3109/09540261.2010.545988

Sandhu, D. S., \& Asrabadi, B. R. (1994). Development of an acculturative stress scale for international students: Preliminary findings. Psychological Reports, 75(1), 435-448.

Tummala-Narra, P., Alegria, M., \& Chen, C.-N. (2012). Perceived discrimination, acculturative stress, and depression among South Asians: Mixed findings. Asian American Journal of Psychology, 3(1), 316. doi:10.1037/a0024661

Uusitalo, L. (2007). Advantages and challenges of Bayesian networks in environmental modelling. Ecological Modelling, 203(3-4), 312-318.

Vehtari, A., Gelman, A., \& Gabry, J. (2017). Practical Bayesian model evaluation using leave-one-out cross-validation and WAIC. Statistics and computing, 27(5), 1413-1432. doi:10.1007/s11222016-9696-4

Vuong, Q.-H. (2018). The (ir)rational consideration of the cost of science in transition economies. Nature Human Behaviour, 2, 5. doi:10.1038/s41562-017-0281-4

Vuong, Q.-H. (2020). Reform retractions to make them more transparent. Nature, 582, 149. doi:10.1038/d41586-020-01694-x

Vuong, Q.-H., Bui, Q.-K., La, V.-P., Vuong, T.-T., Nguyen, V.-H. T., Ho, M.-T., . . Ho, M.-T. (2018). Cultural additivity: behavioural insights from the interaction of Confucianism, Buddhism and Taoism in folktales. Palgrave Communications, 4, 143. doi:10.1057/s41599-018-0189-2 
Vuong, Q.-H., La, V.-P., Nguyen, M.-H., Ho, M.-T., Ho, M.-T., \& Mantello, P. (2020). Improving Bayesian statistics understanding in the age of Big Data with the bayesvl R package. Software Impacts, 4 , 100016. doi:10.1016/j.simpa.2020.100016

Vuong, Q.-H., La, V.-P., Nguyen, M.-H., Ho, M.-T., Tran, T., \& Ho, M.-T. (2020). Bayesian analysis for social data: A step-by-step protocol and interpretation. MethodsX, 7, 100924. doi:10.1016/j.mex.2020.100924

Vuong, Q.-H., Nguyen, M.-H., \& Le, T.-T. (2021a). Home Scholarly Culture, Book Selection Reason, and Academic Performance: Pathways to Book Reading Interest among Secondary School Students. European Journal of Investigation in Health, Psychology and Education, 11(2), 468-495. doi:10.3390/ejihpe11020034

Vuong, Q.-H., Nguyen, M.-H., \& Le, T.-T. (2021b). A mindsponge-based investigation into the psychoreligious mechanism behind suicide attacks. Warsaw: Sciendo.

Vuong, Q. H. (2016). Global Mindset as the Integration of Emerging Socio-Cultural Values Through Mindsponge Processes : A Transition Economy Perspective. In J. Kuada (Ed.), Global Mindsets: Exploration and Perspectives (pp. 109-126). London: Routledge.

Vuong, Q. H., \& Napier, N. K. (2015). Acculturation and global mindsponge: An emerging market perspective. International Journal of Intercultural Relations, 49, 354-367. doi:10.1016/j.ijintrel.2015.06.003

Wagenmakers, E.-J., Marsman, M., Jamil, T., Ly, A., Verhagen, J., Love, J., . . Epskamp, S. (2018). Bayesian inference for psychology. Part I: Theoretical advantages and practical ramifications. Psychonomic bulletin review, 25(1), 35-57.

Westenhoefer, J. (2005). Age and Gender Dependent Profile of Food Choice. In I. Elmadfa (Ed.), Forum of Nutrition (Vol. 57, pp. 44-51). Basel: Karger.

Yeh, C. J., \& Inose, M. (2003). International students' reported English fluency, social support satisfaction, and social connectedness as predictors of acculturative stress. Counselling Psychology Quarterly, 16(1), 15-28. doi:10.1080/0951507031000114058 